Revue

Revue de l'histoire des religions

del'histoire des religions

\title{
Religion, secret et autorité. Pratiques textuelles et cultuelles en clandestinité
}

Avant-propos

\section{Chrystel Bernat et Deborah Puccio-Den}

\section{(2) OpenEdition \\ Journals}

Édition électronique

URL : http://journals.openedition.org/rhr/7764

DOI : $10.4000 /$ rhr.7764

ISSN : 2105-2573

Éditeur

Armand Colin

Édition imprimée

Date de publication : 1 juin 2011

Pagination : 155-161

ISBN : 978-2200-92720-2

ISSN : 0035-1423

Référence électronique

Chrystel Bernat et Deborah Puccio-Den, « Religion, secret et autorité. Pratiques textuelles et cultuelles en clandestinité », Revue de l'histoire des religions [En ligne], 2 | 2011, mis en ligne le 01 juin 2015,

consulté le 22 septembre 2020. URL : http://journals.openedition.org/rhr/7764 ; DOI : https://doi.org/ 10.4000/rhr.7764 


\title{
Religion, secret et autorité Pratiques textuelles et cultuelles en clandestinité
}

\author{
Avant-propos
}

Situé à la croisée d'une foule de thèmes d'étude, le secret a fait l'objet de multiples investigations. Il ne s'agit pas de prétendre reprendre le sujet à nouveaux frais mais d'apporter une contribution au dialogue, dans le cadre d'une approche délibérément pluridisciplinaire avec ce que cela suppose de rencontres et de distanciation, d'affinités méthodologiques et de distinctions épistémologiques. Ce n'est d'ailleurs pas le secret en tant que tel qui nous intéresse mais son articulation spécifique au religieux et ses agencements avec différentes formes d'autorités, scripturaire, doctrinale, rituelle, charismatique. Chacune d'elles revendique une norme, porte des obligations, fixe des interdits et crée l'illicite en postulant une orthodoxie d'où naissent marginalités et minorités.

Tantôt mobilisé en amont de l'élaboration d'une autorité dont il accompagne la quête de légitimité, tantôt utilisé en aval de l'exercice d'un pouvoir dont il valorise l'application et la domination (politique, théologique ou idéologique), le secret épouse des rôles dynamiques contradictoires. Tous ne visent pas une suprématie, tous cependant procèdent d'un rapport de force avec l'autorité instituée, en butte à la protestation d'une existence. Signe manifeste de tensions, souvent liées à une vitalité spirituelle menacée par des prétentions rivales à la Vérité qui caractérisent la culture des religions monothéistes, le recours au secret sert aussi bien l'instauration d'un 
pouvoir que la structuration d'une opposition vis-à-vis d'une autorité ou d'une pensée dominante.

Prenant en considération des minorités - religieuses ou politiques-, des communautés en situation de clandestinité, des élites intellectuelles en opposition avec leur régime ou des sociétés secrètes ayant fait l'objet de poursuites judiciaires, de répression ou de persécution de la part du pouvoir, ce volume propose d'analyser la fonction sociale du secret dans ces univers marqués par un rapport problématique à l'autorité centrale. Ce rapport y est exploré à partir d'une double interrogation: comment se défend une doctrine ou une option confessionnelle, se transmettent des croyances, se maintiennent des entités face à un pouvoir oppressif? Comment émergent de nouvelles figures ou formes d'autorité permettant de garantir une cohésion interne au sein de ces collectivités menacées? Se pose alors la question des modèles, des référents, des procédés et des objets mobilisés pour se soustraire à une domination, à tout le moins pour agencer une marginalité contrainte. À travers quels types de pratiques cachées ou de discours dissimulés, et par le biais de quels usages de l'écrit chacune d'elles cherche-t-elle à s'affranchir d'une tutelle?

Si nous avons principalement interrogé des collectifs contestant au pouvoir institué le monopole du savoir et du sacré, nous avons également exploré l'usage du secret au sein d'instances d'autorités établies - Église catholique, orthodoxie sunnite, pouvoir d'État en Chine, au Maroc et en Italie -, où le savoir ne se distribue pas de façon homogène et transparente mais suscite des retenues et des censures, exhumant le rôle souvent essentiel du secret dans l'affirmation et l'affermissement de l'autorité. Ainsi, tout en se focalisant sur des groupes marqués par leur position minoritaire, notre réflexion met en évidence le fait que la dissimulation ne relève pas d'une certaine catégorie d'hommes - ceux qui se cachent ou ont des choses à cacher - mais appartient aux sociétés humaines, comme l'une de leurs propriétés les plus universellement répandues.

Dans leur pratique de terrain, les anthropologues ont été confrontés d'emblée à la dissimulation. On pourrait affirmer, avec James Clifford (Malaise dans la culture. L'ethnographie, la littérature et l'art au $X X^{e}$ siècle, 1996), que l'ethnologie a été, tout entière, dominée par le paradigme du secret. Avant de devenir un objet théorique, ce dernier s'est trouvé au cœur de la rencontre ethnographique. Si 
Georges Balandier (Afrique ambiguë, 1957) affirme qu'une société se révèle autant à travers ce qu'elle laisse voir, que par la manière dont elle utilise le secret - comme mode d'accès privilégié au monde social -, Andras Zempleni ( Savoir taire. Du secret et de l'intrusion ethnologique dans la vie des autres », Gradhiva, 1996) décrit les formes de dissimulation (du savoir social ou rituel) employées à l'encontre du « professionnel de l'intrusion » qu'est l'ethnologue. Sa réflexion sur « l'économie du secret envisagé comme fait social» met en exergue, au gré d'expériences de terrain menées entre 1976 et 1996, sa «structure feuilletée » découverte à partir des différentes positions que les indigènes lui ont fait occuper. Ce qui rend caduque la définition du secret (du latin secretum) comme quelque chose tenue à l'écart serait « sa tendance incoercible à se frayer une voie vers ses destinataires ». En vertu de cette propriété, détenteurs et destinataires des secrets partageraient tacitement une partie au moins de leur contenu. Ainsi, selon lui, les non-initiés seraient au fait des secrets initiatiques sans avoir le droit de les communiquer. La cohésion du groupe, fondée pour une large part sur "l'interdit de dire ce que l'on sait », en dépendrait.

L'explicitation préalable des termes de cette «convention du silence » qui couvre l'initiation dans la société exotique étudiée par Zampleni, éclaire certains faits sociaux analysés dans ce numéro, comme l'omertà sicilienne ou les tabous linguistiques qui protègent la royauté dans le Maroc contemporain. Il en va du secret mafieux comme du secret initiatique ou du silence enveloppant le pouvoir marocain : ils ne relèvent pas du savoir mais d'une situation d'énonciation et de ses conditions. Il faut suivre l'élaboration théorique d'un autre anthropologue travaillant sur les sociétés initiatiques pour préciser davantage le rapport entre secret et autorité. Dans son ouvrage Les Lois du silence. Essai sur la fonction sociale du secret (1977), Jean Jamin montre que l'initiation n'est pas tant un dispositif apte à produire des différences entre ceux qui savent - les initiés et ceux qui ne savent pas - les non-initiés -, qu'une situation sociale où une catégorie sélective d'individus sont mis en position de « dire ce qu'ils savaient déjà $»$. Le secret n'a donc pas trait au savoir mais au pouvoir, au pouvoir dire, et à l'autorité, au droit de dire. Ce volume explore la possibilité d'élargir ces remarques à d'autres cas d'étude, issus de sociétés anciennes, modernes ou contemporaines, où différents groupes en prise avec le religieux jouent de l'écart 
différentiel entre le pouvoir taire et le pouvoir dire afin de conforter des statuts, consolider des hiérarchies, renforcer des positions d'autorité.

À bien y regarder - soit du fait de la spécificité des sources exploitées par les historiens, soit du fait d'un déplacement théorique que nous avons opéré de la performativité du langage à la performativité de l'écrit-, c'est en définitive davantage le pouvoir écrire et les positions d'autorité qui en découlent qui ont retenu notre attention. On pourrait dire, en citant une nouvelle fois Zampleni (art. cité, 1996), que les matériaux sur lesquels s'appuient les recherches de cet ouvrage sont les secreta, ces sécrétions ou «fragments de secret» que leurs détenteurs, plus ou moins volontairement, laissent échapper. C'est, d'après l'ethnologue, la disposition même du secret qui le conduit fatalement à sa révélation. Au chercheur (« destinataire secondaire » du secret) de repérer ces secreta, d'appréhender ces « signaux » comme autant d' « indices ». Indices de quoi, au juste? De la complexité et de la nature parfois paradoxale du lien que le secret entretient avec l'autorité, qu'il s'agisse de lettres destinées à faire reconnaître l'autorité d'un chef criminel, d'écrits voués à affirmer une foi interdite, de textes préposés à exposer ou divulguer une doctrine ésotérique, de reliques comme supports de pratiques en marge de l'Église revendiquant une médiation privilégiée avec le divin. Plus qu'à signifier la difficulté du secret à rester tel, le fait même que ces sécrétions puissent être constituées en sources par les chercheurs en sciences sociales, ne suggère-t-il pas la nécessité qu'a le secret de se transmettre pour exister et faire exister socialement ceux qui le « sécrètent »?

Reconsidérant l'affirmation selon laquelle toute pratique dissimulatrice réussie ne laisse pas de trace, nous proposons de l'envisager dans ses échappées peu ou prou délibérées et d'approcher les bribes - de textes, de pratiques, de discours et de corps humains - qui forment la matière des études ici rassemblés comme autant de lieux infimes de jeux et d'enjeux de pouvoir qui éprouvent la relation entre secret et autorité. Ce parti pris d'analyser le secret et ses sécrétions comme des produits sociaux qui, inexorablement, se révèlent - sans devoir nécessairement leur révélation à la capacité intrusive des institutions -, conduit à questionner la pertinence de certains schémas du type action/réaction qui se sont imposés suite à la réflexion de Georg Simmel. Dans son ouvrage Secret et sociétés secrètes (trad. 1996), 
le sociologue allemand soutient que ces dernières - qu'il s'agisse de sectes, de groupes ésotériques ou de formes de dissidence religieuseapparaissent en réaction à un pouvoir (policier et judiciaire, étatique ou religieux) de type répressif. Dès lors, les sociétés secrètes sont, au mieux, des formes de "marginalisation active», au pire des « contre-sociétés » où le surgissement du secret est toujours le signe d'un dysfonctionnement. Même si dans certains cas, ici examinés, le secret semble un pis-aller conditionné par la persécution, il ne s'agit pas de limiter les conditions de son recours à un environnement social et politique oppressif. On perdrait à ne considérer que la valeur négative du secret. Simmel lui-même en parle comme d'une «technique sociologique », une "forme de l'action », nous incitant à enquêter sur la dimension pragmatique du secret et sur sa performativité.

Le penseur allemand aborde aussi la question du rapport entre secret et histoire, postulant que les sociétés secrètes sont soit typiques d'un stade archaïque, destiné à être remplacé par des formes nouvelles, soit expressions d'une avant-garde, nécessitant d'être protégée par le secret. Les contributions ici réunies permettent de remettre en question la pertinence d'une théorie évolutionniste du secret. Selon Alain Dewerpe (Espion. Une anthropologie historique du secret d'État contemporain, 1994), rien n'apparaît si peu marqué par l'histoire que le secret, sorte de permanence qui semble défier toute historicisation ou relever d'une temporalité aussi lente qu'imperceptible. Prenant à contre-pied une conception progressiste du secret, Pierre Nora («Simmel : le mot de passe », Nouvelle Revue de Psychanalyse, 1976) se demande quelle est la légitimité du secret et quels sont ses réaménagements dans nos sociétés démocratiques, censées être fondées sur la publicité et sur la transparence. Comment se fait-il que l'Europe du XIX ${ }^{\mathrm{e}}$ siècle ait connu une vague de sociétés secrètes, alors même que la démocratie, dans le sens tocquevillien du terme, s'y installait? L'historien explique que l'avènement du savoir scientifique et la « désacralisation » du pouvoir théocratique issus de l'Ancien régime compliquent la « géographie traditionnelle du secret» qui s'appuyait sur des distinctions (de corps, d'ordres et de classes) anéanties par la démocratisation. C'est en ce qu'elles refusent le secret que les sociétés contemporaines se voient contraintes, voire «condamnées » à le produire. La question soulève celle du rapport entre secret et transparence 
interrogeant les conditions d'exercice du secret dans les mondes anciens, modernes et contemporains. Sans récuser toutes spécificités temporelles, l'un des objectifs de notre réflexion est de saisir une économie du secret.

Pouvoir, savoir et sacré exsudent du secret. L'autorité apparaît comme un dispositif de croyance qui se maintient grâce au secret, couvrant ses ressorts d'un masque (Incidence, 2006). Le topique littéraire nous suggère qu'il faut l'ingénuité d'un enfant pour faire éclater au grand jour la vérité par tous déniée : «Le roi est nu!» (Andersen). Autrement dit, il suffirait qu'une seule voix se lève pour contester à l'autorité son pouvoir de distiller du secret visant à envelopper de sacré ses origines, sa nature, ses rouages. Or, ces entreprises de dévoilement sont coûteuses et d'ordinaire vouées à l'échec. Nombre de cas ici présentés donnent à voir l'immense effort que les instances de pouvoir soutiennent pour maintenir ce « déni de réalité ».

Une première séquence intitulée Persécution, hostilité, clandestinité interroge l'usage du secret dans le cadre de sujétions multiples que les uns cherchent à imposer, les autres à contrecarrer. En un deuxième mouvement relevant plus spécifiquement d'une marque de domination liée à un pouvoir d'interprétation, trois réflexions dissèquent les mises en scène du secret qui, tour à tour, tendent à préserver ou attenter à une autorité politico-religieuse. Chacune de ces contributions discute l'aspect structurant du secret fondé sur un monopole du sens. L'ultime volet, Dissimulation et ontologie des groupes sociaux, sonde la dimension métaphysique du secret. La configuration thématique du volume qui vise à dégager les principaux cadres d'analyse de l'enquête ne doit pas masquer l'entrecroisement des recherches et le foisonnement de thèmes transversaux que soulève le thème du secret. C'est ici leurs déclinaisons en des systèmes de pensée et de croyances variés sinon concurrentiels qui nous intéressent.

Ce numéro thématique est le fruit d'une réflexion comparative engagée, de 2005 à 2007, entre chercheurs de disciplines connexes - histoire et histoire de la philosophie, anthropologie, ethnologie et sociologie - dans le cadre d'un séminaire de recherche tenu par nos 
soins à l'École Pratique des Hautes Études (Section des sciences religieuses) et affilié au Centre d'études des religions du Livre. Il est en partie issu d'une journée d'étude qui exposa cette enquête collective, d'abord consacrée aux relations entre «textes et autorité » puis à celles de " religion, secret et autorité », organisée en juin 2008 avec le soutien des membres de l'École doctorale 472 et du Laboratoire d'études sur les Monothéismes (UMR 8584 CNRS/EPHE/Université de Paris IV-Sorbonne/Université de SaintÉtienne), auxquels nous adressons nos plus vifs remerciements. Remaniée, l'édition a incorporé trois contributions qui n'étaient pas incluses dans le programme de la journée d'étude initiale; celles de Asma Hilali (Institute of Ismaili Studies, Londres), de Francesca Sbardella (Université de Bologne) et de Frédéric Gabriel (CNRS Institut d'histoire de la pensée classique). À chacun, nous exprimons notre gratitude.

chrystel.bernat@ephe.sorbonne.fr deborah.puccio-den@ehess.fr 